\title{
Kainella Walcott, 1925 (Trilobita, Ordovícico Temprano) en el noroeste de Argentina y sur de Bolivia. Importancia bioestratigráfica
}

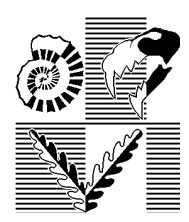

\author{
N. Emilio VACCARI ${ }^{1}$, Beatriz G. WAISFELD ${ }^{1}$, Larisa F. MARENGOำ y LeGrand SMITH²
}

\begin{abstract}
Kainella Walcott (Trilobita, early Ordovician) in Northwestern Argentina and Southern BOLIVIA. BIOSTRATIGRAPHIC SIGNIFICANCE. The Tremadocian Kainella meridionalis Zone in the Central Andean Basin is is reassessed. This zone has been widely used for regional and intercontinental correlations, but such correlations mostly involved an extended interval that corresponds, in fact, to the whole range of the genus. Species of Kainella recorded in northwestern Argentina and southern Bolivia exhibit a relatively wide geographic distribution and a constrained stratigraphic range. On the basis of their record a succession of biozones is proposed, ranging from the basal Tremadocian to possibly the early middle Tremadocian. Three biozones are proposed on the basis of integrated information drawn from several localities, i.e., the K. andina, K. meridionalis, and K. teiichii zones. In addition, the Jujuyaspis keideli Subzone is here upgraded to the rank of zone. The definition of this succession of biozones is a significant progress in the establishment of a high resolution biostratigraphic framework based on trilobites, leading to more precise correlations in the future.

Resumen. Se llevó a cabo una revisión de la Biozona de Kainella meridionalis del Tremadociano de la Cuenca Andina Central. La Biozona de K. meridionalis ha sido utilizada para establecer correlaciones a escala regional e intercontinental. Sin embargo, de acuerdo a esta revisión, dichas correlaciones han tomado un intervalo amplio que corresponde, en realidad, al rango completo del género en la región. Las especies de Kainella registradas en el noroeste de Argentina y sur de Bolivia tienen una amplia distribución geográfica y acotado rango estratigráfico. En base a su registro se realiza una propuesta de biozonas, cuya sucesión abarca desde el Tremadociano basal hasta, posiblemente, el Tremadociano medio bajo. Integrando información de numerosas localidades, se propone aquí la sucesión de las siguientes biozonas: Biozona de K. andina, Biozona de K. meridionalis y Biozona de K. teiichii. Además, se eleva al rango de Biozona la Subzona de Jujuyaspis keideli. La definición de esta sucesión de biozonas representa un progreso significativo en el establecimiento de un esquema bioestratigráfico de alta resolución en base a trilobites, fundamental para establecer correlaciones más precisas en el futuro.
\end{abstract}

Key words. Trilobita. Kainella. Ordovician. Northwestern Argentina. Southern Bolivia. Biostratigraphy. Correlation.

Palabras clave. Trilobita. Kainella. Ordovícico. Noroeste de Argentina. Sur de Bolivia. Bioestratigrafía. Correlación.

\section{Introducción}

El género Kainella Walcott, 1925, tiene una amplia distribución geográfica y sus especies han sido registradas en América del Norte, América del Sur y Asia. Debido a que este género tiene un rango estratigráfico relativamente corto, sus especies han sido utilizadas como indicadores bioestratigráficos, tanto en correlaciones regionales como intercontinentales.

${ }^{1}$ CICTERRA, CIPAL (Centro de Investigaciones Paleobiológicas, FCEFyN Universidad Nacional de Córdoba, Av. Vélez Sárfield 299, 5000 Córdoba, Argentina. evaccari@efn.uncor.edu

2266 Merrimon Avenue, 16, Asheville, North Carolina 28801, USA

(c)Asociación Paleontológica Argentina
Kainella es un taxón frecuente en América del Sur, con una distribución relativamente amplia en diferentes unidades de la Cordillera Oriental Argentina, y en su extensión hacia el norte, el segmento Yunchará en Bolivia. La especie más frecuentemente citada en la región, Kainella meridionalis Kobayashi, fue definida sobre la base de material procedente de la localidad Pascha-Incamayo (Kobashayi, 1935). Kobayashi (1937) fue el primero en reconocer, mediante comparación con especies del género descriptas en distintas localidades de América del Norte, el valor bioestratigráfico de K. meridionalis. Este autor designó como "Fauna de Kainella" a la asociación de trilobites que corrientemente acompañan a K. meridionalis en la localidad de Pascha. Agunos de ellos son, entre otros, Asaphellus catamarcensis Kobayashi, 1937, "Kainella" conica Kobayashi, 1935 y Leptoplastides ar- 
gentinensis (Kobayashi, 1935). Esta denominación fue empleada por Harrington (1938), quien incluyó en la Fauna de Kainella elementos adicionales procedentes de la Quebrada del Toro y de la región de Azul Pampa, refiriendo la mencionada fauna al Tremadociano inferior. Posteriormente, (Harrington y Leanza, 1957) definieron la Biozona de Kainella meridionalis en base a material de numerosas localidades del noroeste argentino.

En este trabajo presentamos una revisión de la Biozona de Kainella meridionalis. La misma se sustenta en un detallado estudio taxonómico de las especies de Kainella de Argentina y Bolivia, sobre la base de nuevo material colectado en ambos países (Vaccari y Waisfeld, este volumen). Dicho estudio reveló una diversidad significativamente mayor que la previamente conocida, e involucró la revisión de la especie nominal de la biozona, la caracterización de cuatro nuevas especies (K. teiichii, K. morena, Kainella sp. nov. A y Kainella sp. nov. B), y la redescripción de $K$. andina Suarez Soruco, así como un relevamiento exhaustivo de la distribución geográfica y estratigráfica de cada una de ellas. Sobre la base de esta información se propone un nuevo esquema bioestratigráfico regional, se establecen nuevas correlaciones a escala de la cuenca, y se reevalúan otras a escala intercontinental. Además, en base a colecciones realizadas en las localidades de Steimmann y Hoek (Cuesta de Erquis y Tambo Guanacuno) en Bolivia, se aportan nuevos datos referidos a la edad de las mismas.

Estudios recientes han demostrado que la historia depositacional de las unidades tremadocianas de la Cordillera Oriental está caracterizada por la superposición de eventos de signatura tanto eustática como tectónica (Astini, 2003, 2005; Buatois et al., 2006). Esto da como resultado un complejo apilamiento de facies y frecuentes hiatos estratigráficos, que sólo pueden ser resueltos contando con un contexto bioestratigráfico de alta resolución. La distribución espacio-temporal de las especies de Kainella que se presenta en esta contribución, constituye un progreso significativo en el establecimiento de un esquema bioestratigráfico de dichas características, permitiendo una correlación más precisa que aquella alcanzada por estudios previos.

\section{Estratigrafía}

Las especies de Kainella en territorio argentino que se analizan en este trabajo se distribuyen en diferentes unidades tremadocianas de la Cordillera Oriental. Estas unidades presentan una manifiesta complejidad tanto desde el punto de vista estratigráfico y paleoambiental como estructural. Esta complejidad es responsable en parte de la variada nomenAMEGHINIANA 47 (3), 2010 clatura que han recibido las unidades litoestratigráficas tremadocianas a través de la cuenca. En este trabajo, y hasta tanto se alcance un consenso para una nomenclatura unificada con sentido estratigráfico, se ha preferido mantener los nombres locales de las unidades tremadocianas. Usualmente, estas unidades se incluyen en forma general dentro de la Formación Santa Rosita (Cámbrico Tardío-Tremadociano), que está representada por una compleja sucesión de ambientes marinos relativamente someros, que varían desde plataforma distal a shoreface, con transiciones a ambientes deltaicos, estuarinos y fluviales (Astini, 2003; Buatois y Mángano, 2003; Buatois et al., 2006).

El conocimiento estratigráfico y paleontológico de las distintas unidades portadoras de Kainella que se abordan en este trabajo es muy dispar. Un análisis detallado de éstas escapa a los objetivos de este trabajo. Con el fin de brindar un marco general al registro de las distintas especies y al esquema que se propone aquí, a continuación se realiza una breve síntesis de la ubicación geográfica y estratigráfica de los taxones que se mencionan (figuras 1 y 2).

En la región de Pascha-Incamayo (Quebrada del Toro, $40 \mathrm{~km}$ al noroeste de Salta), se registran tres especies de Kainella: K. meridionalis, K. teiichii, y K. cf. K. teiichii. El único ejemplar de nuestra colección referido a K. meridionalis Kobayashi proviene de un clasto dentro de los flujos de detritos de la Formación Cardonal, mientras que $K$. cf. $K$. teiichii fue registrada en un bloque dentro de los conglomerados de la Formación Devendeus. Ambas unidades fueron descriptas e interpretadas desde el punto de vista estratigráfico y paleoambiental por Astini (2003). Según este autor, la Formación Cardonal es una unidad heterogénea constituida por bancos de areniscas de escala métrica, cuerpos lenticulares de flujos de detritos conteniendo frecuentes clastos portadores de fauna marina y cuerpos conglomerádicos. Esta unidad fue interpretada como característica de ambientes con dominio mareal. La Formación Devendeus está compuesta por un conjunto predominantemente conglomerádico caracterizado por clastos de variada composición con un contenido faunístico muy diverso. Según Astini (2003), la Formación Devendeus puede ser interpretada como el resultado de sucesiones estratigráficas erosionadas y redepositadas, implicando una importante discontinuidad. La procedencia del material tipo de $K$. meridionalis no ha podido ser precisada, más aún teniendo en cuenta la complicada sucesión de unidades de la región y la variada procedencia de los bloques que contienen las formaciones Cardonal y Devendeus. Las contribuciones de Rao y Tortello (1998), Rubinstein et al. (1999), Tortello y Rao (2000), Ortega y Albanesi (2002, 2003); Astini (2005), Waisfeld et al. (2006), Waisfeld y Vaccari (2003; 2006), Benedetto (2007), Salas et al. (2007) y Toro et al. (2010), 


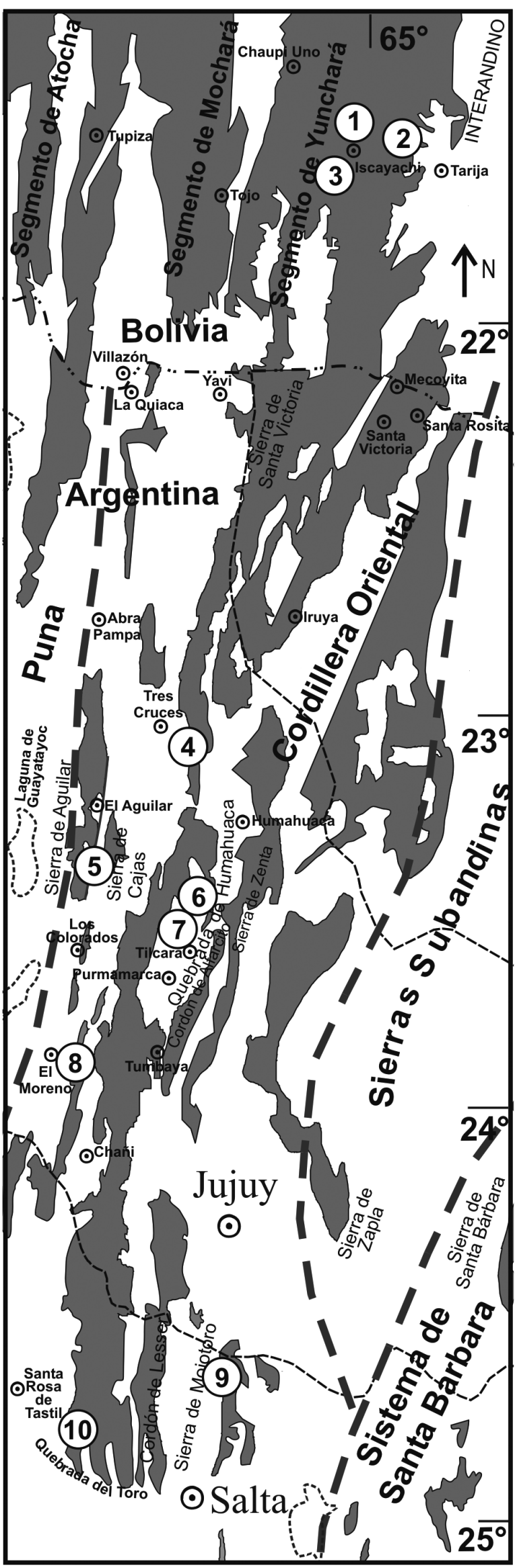

aportan nuevos datos paleontológicos, estratigráficos y bioestratigráficos para la región.

K. meridionalis Kobayashi, procede del Miembro Alfarcito y parte basal del Miembro Rupasca y K. teiichii del Miembro Rupasca de la Formación Santa Rosita, expuesta en Quebrada Arenal, tributaria del Río Grande, en las proximidades de la localidad de Perchel. Los miembros Alfarcito y Rupasca fueron estudiados en detalle desde el punto de vista sedimentológico en localidades próximas de la Quebrada de Humahuaca (área de Alfarcito, Tilcara) por Buatois et al. (2006). En el Miembro Alfarcito, estos autores reconocieron tres ciclos grano y estrato crecientes de extensión regional en la Quebrada de Humahuaca. Al mismo tiempo, interpretaron los intervalos arenosos como depósitos de shoreface inferior a medio, y los intervalos heterolíticos de grano fino como offshore inferior - transición a offshore. En la sección de Quebrada Arenal se reconocen parcialmente los ciclos descriptos por estos autores, registrándose $K$. meridionalis en la mitad superior de la unidad. De acuerdo a Buatois et al. (2006), el Miembro Rupasca corresponde a un episodio transgresivo cuyo tramo inferior consiste en depósitos de offshore inferior/transición a offshore, y el superior a depósitos de plataforma a offshore inferior. En Quebrada Arenal se reconoce el tramo inferior del Miembro Rupasca donde se registra K. teiichii (figura 2).

K. meridionalis Kobayashi se registra también en la Formación Caldera expuesta en las proximidades de la localidad de La Caldera, sobre la Ruta Nacional 9. Esta unidad está compuesta por bancos relativamente potentes de areniscas con estratificación cruzada hummocky, frecuentemente amalgamados, y es portadora de concentraciones fosilíferas dominadas por trilobites y braquiópodos rhynchonelliformes. Esta sucesión fue interpretada por Moya $(1988 ; 1998)$ como depósitos de tormentas en un ambiente de shoreface. No se cuenta con estudios paleontológicos de esta localidad, excepto por el registro de un único braquiópodo (Benedetto, 2007).

Kainella sp. nov. A y Kainella sp. se registran en el Miembro Alfarcito en la Quebrada Trancas (ubicada $300 \mathrm{~m}$ al norte de la Quebrada Arenal). Kainella sp. nov. A se registra también en el Miembro Alfarcito en la Quebrada de Moya, en las cercanías del Angosto de Chucalezna. Información estratigráfica y paleontológica sobre la sucesión expuesta en esta lo-

Figura 1. Mapa de ubicación (modificado de Astini, 2003 y Müller et al., 1996). Los números corresponden a la procedencia de las especies de Kainella mencionadas en el texto / location map (modified from Astini, 2003, and Müller et al., 1996). Numbers indicate localities where Kainella species mentioned in the text were recorded. 1, Tambo Guanacuno, 2, Erquis, 3, Palqui, 4, Azul Pampa; 5, Sierra de Cajas, 6, Quebrada Moya, 7, Quebrada Arenal, 8, El Moreno, 9, La Caldera, 10, Pascha. 


\section{Quebrada Arenal}

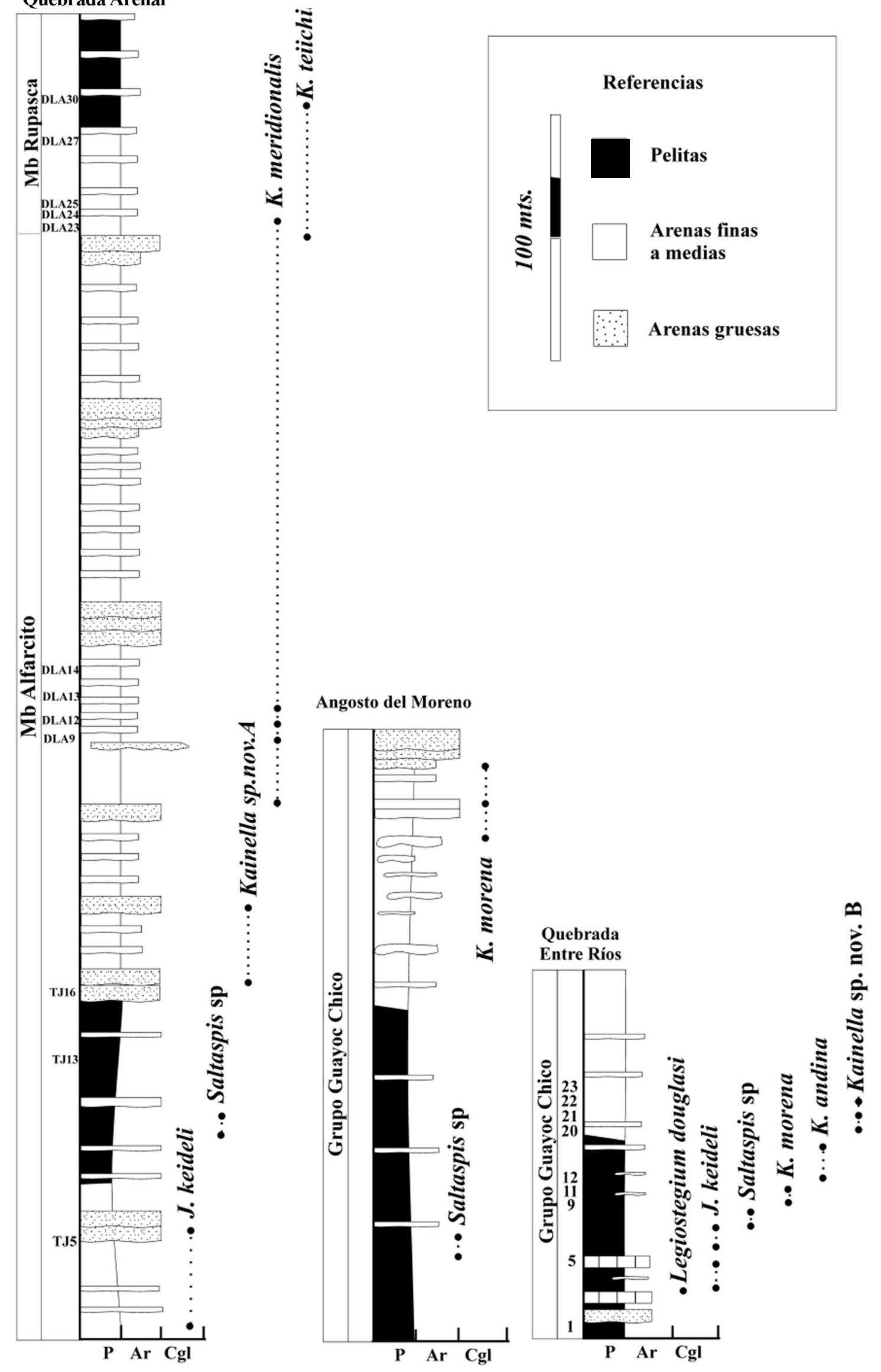


calidad pueden encontrarse en Buatois y Mángano (2003), Mángano y Buatois (2004), Albanesi y Aceñolaza (2005), y Vergel et al. (2007) (ver figura 1).

Kainella morena proviene del Grupo Guayoc Chico (sensu Astini, 2003), en la localidad de Angosto del Moreno, ubicada en las proximidades de El Moreno (figura 1). K. morena se registra en el miembro superior de coquinas de Astini (2003) o tramo superior del intervalo o unidad 4 de Moya et al. (2003) y Buatois et al. (2003). Este miembro fue interpretado como depósitos de shoreface. Además de las contribuciones mencionadas, puede encontrarse información estratigráfica y paleontológica adicional en Moya y Monteros (2000), Moya y Albanesi (2000), Gómez Martínez et al. (2002), Ortega y Albanesi (2005) y Benedetto (2007).

K. andina, K. morena y Kainella nov. sp. B se registran en la sección de Entre Ríos, en el flanco oriental de la Sierra de Cajas (figura 1), donde se ha podido documentar con precisión el rango de estas especies. La Sierra de Cajas constituye un anticlinal fallado, la mayoría de las secciones medidas se encuentran en su extremo norte, coincidiendo en parte con el cierre periclinal. En ninguna de las secciones medidas se observa la sucesión estratigráfica completa, lo que ha generado algunas interpretaciones erróneas. Aceñolaza (1968) reconoció en el área de la Sierra de Cajas la presencia del las formaciones Padrioc, Lampazar, Cardonal, Acoite y Sepulturas. Méndez (1973) siguió la nomenclatura propuesta por Turner para la Sierra de Santa Victoria, identificando las formaciones Santa Rosita y Acoite. Benedetto (1977) reconoció como Formación Santa Rosita a las pelitas con intercalaciones de areniscas finas y lentes de calizas de la Biozona de Neoparabolina frequens argentina y como Formación Cardonal a las areniscas y coquinas con $K$. meridionalis. Rao y Hünicken (1995) presentaron dos secciones; en la primera (Quebrada La Vizcacha) indicaron la distribución de conodontes de la Formación Lampazar y en la otra (Quebrada Amarilla) asignaron la parte inferior de la sección a la Formación Lampazar y el tramo superior a la Formación Cardonal. Más tarde, Tortello et al. (1999) incluyeron toda la columna estratigráfica de esta última sección en la Formación Cardonal. Existe algo más de consenso entre los diferentes autores respecto de la sección de Quebrada Azul, donde la mayoría ha asignado las rocas allí expuestas a la Formación Lampazar (Tortello y Aceñolaza, 1993; Tortello y Esteban, 2003). Dos secciones fueron relevadas en el área durante el presente estudio. Una corresponde a la Quebrada de la Vizcacha y la otra más al sur en el Paraje Entre Ríos, en el flanco oriental de la Sierra de Cajas. En la primera sección, sobre las cuarcitas de la Formación Padrioc, se apoya la Formación Lampazar constituida por una sucesión de lutitas que culmina con cuarcitas blanquecinas. En toda la columna se registra la presencia de $N$. frequens argentina (Kayser). En la sección medida más al sur, en contacto tectónico con las pelitas de la Formación Lampazar, portadora de la fauna de $N$. frequens argentina, yace una sucesión de pelitas con intercalaciones calcáreas y areniscas subordinadas, donde se ha podido corroborar la presencia de Jujuyaspis keideli Kobayashi y Rhabdinopora Eichwald. En esta secuencia se intercalan niveles de coquinas portadoras de las especies de Kainella (figura 2). Si bien esta sucesión fue referida a la Formación Cardonal por otros autores, se prefiere no emplear esta denominación debido a las significativas diferencias litofaciales con esa unidad tal como está expuesta en su localidad tipo (área de PaschaIncamayo, cf. Astini, 2003). Hasta tanto se realice una revisión detallada de la estratigrafía de la Sierra de Cajas, se empleará en forma preliminar la denominación de Grupo Guayoc Chico, en virtud de que esta sucesión exhibe ciertas similitudes con aquélla que suprayace a la Formación Lampazar en la sección del Angosto del Moreno. Descripciones de las unidades expuestas en el área y su contenido paleontológico pueden encontrarse en los trabajos de Aceñolaza (1968), Rao y Hünicken (1995), Rao (1999), Tortello et al. (1999), Tortello y Esteban (2003), Esteban y Tortello (2007) y Benedetto (2007), entre otros.

Kainella andina y K. morena también se registran en las proximidades de la localidad de Azul Pampa, un kilómetro al oeste de la estación de ferrocarril (figura 1). El material fue colectado de cuerpos de areniscas rojas y rosadas que caracterizan los niveles de transición entre las formaciones Casayok y Azul Pampa (Harrington y Leanza, 1957; Fernández, 1985) y de bancos de coquinas producto de eventos tempestíticos (Benedetto, 2007).

Las localidades bolivianas analizadas en este trabajo se encuentran ubicadas en el Segmento Yunchará (Erdtmann et al., 1995), que corresponde a la prolongación norte de la Cordillera Oriental. Aquí aflora extensamente la Formación Iscayachi (Cámbrico Superior-Tremadociano inferior). Esta unidad, de un espesor aproximado de 1200 a 1500 m (Erdtmann et al., 1995), forma sucesiones homoclinales suavemente inclinadas. Sin embargo, internamente, los fósiles evidencian una importante deformación.

Figura 2. Columnas estratigráficas de las secciones de Quebrada Arenal, Angosto del Moreno y Sierra de Cajas (Quebrada Entre Ríos) y distribución vertical de las especies de trilobites / stratigraphic sections at the Quebrada Arenal, Angosto del Moreno, and Sierra de Cajas (Quebrada Entre Ríos), showing vertical distribution of trilobites species. 
La Formación Iscayachi está compuesta por una alternancia de areniscas de grano fino y limolitas grises, con superficies de alteración de color ocre. En los planos de laminación se encuentran acumulaciones de fósiles formando pavimentos. Estas sucesiones han sido interpretadas como habiéndose formado en un prodelta influenciado por tormentas o incluso hasta en un frente deltaico (Egenhoff et al., 2004).

La localidad tipo de $K$. andina se encuentra sobre un camino de herradura denominado Cuesta de Erquis, que une la localidad de Erquis con la ruta Tarija-Villazón, aproximadamente $10 \mathrm{~km}$ al noroeste de Tarija (figura 1). La localidad de Tambo Guanacuno se encuentra $12 \mathrm{~km}$ al nor-noroeste de Iscayachi, por la ruta que une Iscayachi-Camargo. Tanto Cuesta de Erquis como Tambo Guanacuno son localidades clásicas del trabajo pionero sobre las faunas del Paleozoico Inferior de Bolivia realizado por Steinmann y Hoek (1912), que fueran posteriormente redescriptas por Kobayashi (1937). La Quebrada de Palqui se encuentra en la provincia de Aviléz, al sudoeste de Iscayachi y se accede a ella desde un desvío hacia el sur de la ruta que une Iscayachi-Villazón pasando el Abra de Chorcoya (Suárez Soruco, 1975) (figura 1).

\section{Bioestratigrafía}

Reevaluación de la Biozona de Kainella meridionalis

Harrington y Leanza (1957) definieron la Biozona de Kainella meridionalis para el Tremadociano inferior, en base a extensas colecciones procedentes de numerosas localidades del noroeste argentino. Estos autores mencionaron el registro de K. meridionalis en el área de Pascha-Incamayo (Angosto La Quesera, Angosto de Pascha, Quebrada Lampazar), región de Tilcara (Quebrada Rupasca y Quebrada San Gregorio), norte (Azul Pampa) y oeste de la Quebrada de Humahuaca (Río Yacoraite y Quebrada Huichaira), Sierra de Mojotoro (área de La Caldera) y región de Santa Victoria-Iruya (Quebrada Colorada, Nazareno, Río Bocoyá, Río Santa Victoria). Autores posteriores extendieron aún más su registro, a las áreas de Sierra de Cajas (Quebrada Amarilla, Tortello et al., 1999), Angosto del Moreno (Moya y Albanesi, 2000) y Abra de Zenta (Aceñolaza et al., (2003). Además, se llevaron a cabo diversos intentos de calibrar su rango estratigráfico a partir de elementos como graptolitos y conodontes, y fueron propuestos diversos esquemas de correlación (e.g., Moya et al., 1994; Zeballo et al. 2005, 2008; Ortega y Albanesi, 2005; Tortello et al. 2002; Benedetto, 2005, 2007, entre otros)

El estudio de Vaccari y Waisfeld (2010), basado en la comparación con el material tipo ilustrado por Kobayashi (1935) y en una rica colección de especíAMEGHINIANA 47 (3), 2010 menes de numerosas localidades, nos permite conocer en detalle la morfología de K. meridionalis precisando de esta forma su distribución espacio-temporal con respecto a la distribución previamente supuesta. Asimismo, formas antes referidas a K. meridionalis son ahora reubicadas en otras especies.

Rao y Tortello (1998), Tortello y Rao (2000) y Ortega y Albanesi $(2002,2003)$ aportaron datos para precisar la edad de la Biozona de K. meridionalis registrada en el área de Pascha-Incamayo, sobre la base de la fauna asociada de conodontes y graptolitos. Tortello y Rao (2000) extendieron el rango de K. meridionalis y de la biozona epónima al tramo medio-superior de la Formación Cardonal y al tramo inferior de la Formación Saladillo. Estos autores indicaron que la parte inferior de la Biozona de K. meridionalis corresponde al Tremadociano medio (parte alta de la Biozona de Cordylodus angulatus), mientras que la parte superior está asociada a conodontes de edad tremadociana tardía-temprana. Ortega y Albanesi (2002, 2003) refirieron el tramo superior de la Biozona de $K$. meridionalis (documentada por Tortello y Rao, 2000) a la Biozona de Bryograptus (Tremadociano tardío-temprano) presente en la parte basal de la Formación Saladillo.

Waisfeld y Vaccari (2003) documentaron que, en el área de Pascha-Incamayo, K. meridionalis y la "Fauna de Kainella" en su totalidad se registran en bloques retrabajados en la base de la Formación Cardonal y en la Formación Devendeus. Hasta el momento, sólo en la base de la Formación Cardonal se han registrado bloques que contuvieran K. meridionalis. Si bien no es posible precisar la edad de esta unidad, la misma estaría restringida a un intervalo muy acotado entre el Tremadociano temprano (por la presencia de $K$. meridionalis) y el Tremadociano medio bajo por la presencia de Bryograptus en la base de la Formación Saladillo (cf. Ortega y Albanesi, 2002). Los bloques de la Formación Devendeus contienen diferentes asociaciones de trilobites, que sugieren un rango de edad amplio para su procedencia, entre el Tremadociano temprano (Fauna de Kainella) y el Tremadociano tardío- Floiano basal?, con lo cual su edad debe ser más joven que la de estos bloques.

Debido a la compleja historia depositacional de las unidades portadoras de K. meridionalis en el área de Pascha-Incamayo y al amplio rango de variación en la morfología de las especies asignadas corrientemente a $K$. meridionalis en la literatura, Waisfeld y Vaccari (2003) pusieron en duda la validez de esta biozona. De acuerdo a los nuevos datos aportados en este trabajo, la presencia de K. meridionalis en el Miembro Alfarcito expuesto en la Quebrada Arenal en una sucesión normal sin retrabajo, permite finalmente establecer su posición estratigráfica con certeza. Si bien no se cuenta aún con datos como para es- 
tablecer su edad con precisión, y teniendo en cuenta la propuesta de Bergström et al. (2009) de tripartición del Tremadociano, su rango se extendería entre el tramo más superior del Tremadociano inferior (Tr1) y la base del Tremadociano medio (Tr2).

En Cuesta de Erquis ha sido mencionada la presencia de K. meridionalis asociada a K. andina (Suárez Soruco, 1975, p. 135). Sin embargo, en nuestras colecciones de los mismos niveles no ha sido posible corroborar la presencia de K. meridionalis tal como fuera revisada por Vaccari y Waisfeld (2010), y sólo se verifica en esta localidad la presencia de K. andina. Asimismo, en esta localidad ha sido mencionada la presencia de Rhabdinopora flabelliformis s.l. (Branisa, 1965). Tomando en consideración el registro de K. andina en la Cordillera Oriental Argentina, en el Grupo Guayoc Chico aflorante en la Sierra de Cajas, es posible establecer una correlación con los niveles de la Formación Iscayachi, portadores de esta especie en Cuesta de Erquis y referirla al Tremadociano inferior.

Suárez Soruco (1975) describió una novedosa fauna de la Formación Iscayachi en la quebrada de Palqui y la correlacionó con los niveles de la Formación Iscayachi de la localidad Cuesta de Erquis, asignándolos todos a la Biozona de K. meridionalis. En la localidad de Tambo Guanacuno, Vaccari et al. (2006) reconocieron en la Formación Iscayachi al menos dos asociaciones de trilobites. La más baja está compuesta por Onychopyge branisai Suárez Soruco, Palquiella incaica Suárez Soruco, Pharostomina alvarezi Suarez Soruco, Asaphelus palquiensis Suárez Soruco, Altiplanelaspis palquiensis Přibyl y Vaněk, 1980, Leptoplastides sp. y Conophrys sp. y la superior compuesta, entre otros, por Saltaspis steinmanni Kobayashi; Parabolinella sp. nov., asociada a los primeros registros de Rhabdinopora spp. Asimismo, en ninguna de estas asociaciones hemos registrado la presencia de Kainella. La asociación inferior tiene la misma composición faunística que la descripta por Suárez Soruco (1975) en la localidad de Palqui y, por lo tanto, son aquí correlacionadas. Si bien no hay elementos precisos para la determinación de la edad de esta asociación, las mismas se encuentran en Tambo Guanacuno por debajo de los primeros registros de Rhabdinopora y por encima de unos niveles de areniscas verdes claras donde Kobayashi (1937) y Branisa $(1965$, p. 226) registraron la presencia de "Plethometupus" microphtalmus y N. frequens argentina respectivamente. En nuestras colecciones de la localidad de Ronqui Angosto, en Argentina, estas dos especies se encuentran en la parte alta de la Biozona de $N$. frequens argentina, $80 \mathrm{~m}$ por debajo del primer registro de J. keideli Kobayashi. Por lo tanto, la asociación más baja de Tambo Guanacuno se ubicaría en la zona del límite Cámbrico-Ordovícico.

Por lo expuesto aquí la localidad de Steinmann y
Hoek de Cuesta de Erquis corresponde al Tremadociano inferior bajo, mientras que la sección de Tambo Guanacuno abarcaría desde el Cámbrico Superior alto al Tremadociano inferior, refiriéndose así la fauna proveniente de Quebrada de Palqui descripta por Suárez Soruco (1975) a la transición Cámbrico-Ordovícico.

\section{Propuesta de nuevo esquema bioestratigráfico para el Tremadociano inferior}

Los trilobites constituyen el componente dominante entre los fósiles del Tremadociano de la Cuenca Andina Central. Por ello, un esquema bioestratigráfico local sobre la base de la distribución de especies de trilobites, se constituye en una herramienta indispensable para entender la complejidad de eventos acaecidos durante el relleno de esta cuenca, la cual se desarrolló sobre un margen continental activo, con varios depocentros y un complejo mosaico de ambientes sedimentarios. La amplia distribución geográfica y el acotado rango estratigráfico que presentan las especies de Kainella aquí estudiadas las convierte en un elemento ideal para proponer un esquema bioestratigráfico regional de alta resolución (figuras 3-5).

Se proponen aquí cuatro biozonas de intervalo para el Tremadociano temprano. Las especies nominales de las biozonas están asociadas a una variada fauna de trilobites, que incluye especies de agnóstidos, leiostégidos, shumárdidos, richardsonéllidos, ceratopygidos, olénidos, asáphidos y ptychopáridos. El estudio sistemático de las mismas se encuentra actualmente en progreso.

Biozona de Jujuyaspis keideli (Tortello et al., 2002; nom. transl.). Esta biozona fue propuesta previamente por Tortello et al. (2002) como subzona superior de la Biozona de Neoparabolina frequens argentina. Su estratotipo ha sido determinado en la Formación Volcancito, en Famatina. Se propone aquí elevar el rango de la misma a Biozona. Esto se fundamenta, esencialmente, en que las faunas asociadas, si bien tienen algunos elementos en común, son claramente distintas. Por otra parte, en varias localidades de la Cuenca Andina Central se registra una discontinuidad estratigráfica de singular magnitud, mediando entre las biozonas de N. frequens argentina y J. keideli (e.g., Arenal-Trancas, Purmamarca, Vaccari et al., 2008). La Biozona de J. keideli se distribuye ampliamente en la Cuenca Andina Central como así también en la Formación Volcancito en Famatina (Tortello et al. 2002, p. 136). Además la presencia de esta especie, permite reconcerla en Noruega (Nikolaisen y Henningsmoen, 1985), Colombia (Baldis et al., 1984) y Bolivia (Přibyl y Vaněk, 1980). Asimismo, esta biozona permite una amplia correlación intercontinental, 


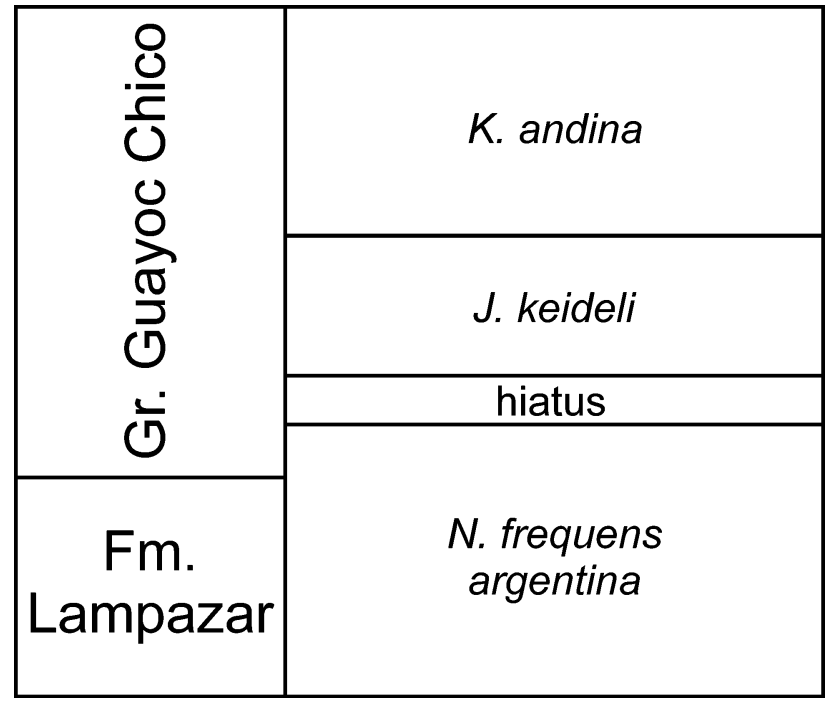

Figura 3. Biozonas reconocidas en el sector occidental de la Cordillera Oriental (Sierra de Cajas-Angosto del Moreno) / biozones recognized in the western area of the Cordillera Oriental (Sierra de Cajas-Angosto del Moreno).

ya que se ha señalado el registro de Jujuyaspis como indicador de la base del Ordovícico (Aceñolaza y Aceñolaza, 1992; Cooper et al., 2001). Recientemente, Zeballo y Albanesi (2008, 2009) determinaron una edad cámbrica tardía (Biozona de Cordylodus intermedius, Subzona de Hirsutodontus simplex) para el registro de J. keideli en su localidad tipo sobre la base del registro de $H$. simplex (Druce y Jones). Si bien en algunas localidades esta especie de conodonte se restringe al Furongiano (e.g., Utah y Texas; Miller et al., 2006), en la sección de Green Point (Terranova), donde ha sido establecida la base del Sistema Ordovícico, su registro completo se encuentra por encima del primer registro de Iapetognatus fluctivagus Nikoll et al., un indicador de la base del Sistema (Barnes, 1988; Cooper et al., 2001). Por ello, la propuesta de una edad cámbrica tardía para J. keideli sobre la base de su asociación con el conodonte $H$. simplex no resulta convincente. Por otra parte, en nuestras colecciones de esta localidad, asociada a J. keideli se registra la presencia de graptolitos (actualmente en estudio) que indicarían una edad no más antigua que tremadociana basal. Para una completa distribución de J. keideli ver Tortello y Clarkson (2003, p. 258).

Biozona de Kainella andina nov. La Biozona de $K$. andina está definida por el primer registro de K. andina en el Grupo Guayoc Chico en la sección de Entre Ríos (Sierra de Cajas), la cual es propuesta como estratotipo de esta biozona. En esta sección, K. andina tiene su primer registro $15 \mathrm{~m}$ por encima del último registro de J. keideli Kobayashi (figuras 2, 3). En la Quebrada Amarilla se reconoce la presencia de K. andina (K. "meridionalis" sensu Tortello et al., 1999) dentro de la Zona de C. angulatus determinada por Rao y AMEGHINIANA 47 (3), 2010
Hünicken (1995). El registro de K. andina en esta sección también se asocia a la presencia de Anisograptus matanensis junto a formas referidas como Rhabdinopora flabelliformis cf. anglica (Ortega y Albanesi, 2005). Esta biozona se reconoce además en las localidades de Azul Pampa, y de Erquis en Bolivia. La misma se refiere al Tremadociano inferior, parte superior del Tr1 de acuerdo al esquema de Bergström et al. (2009) .

La biozona es reconocida también por el registro de K. morena en la sección de Angosto del Moreno. En dicha localidad se documentó la presencia K. morena junto a una asociación de conodontes de baja diversidad, compuesta por Cordylodus angulatus Pander, Monocostatus sevierensis Miller, Teridontus nakamurai (Nogami) y T. obesus Ji y Barnes (Moya et al., 2003) que permitió referir estos estratos a la Zona de Cordylodus angulatus (Tremadociano inferior, parte superior de Tr1 de Bergstöm et al., 2009). Asimismo, Moya et al. (2003) reconocieron los graptolitos Anisograptus matanensis y Rhabdinopora flabelliformis, que se extienden a través de toda la unidad.

Biozona de Kainella meridionalis (redefinida). La base de la Biozona de $K$. meridionalis está definida por el primer registro de esta especie. Se propone la sección de Quebrada Arenal como estratotipo de la misma (figura 4). Su límite superior está dado por el primer registro de K. teiichii en dicha sección. En esta localidad se ha registrado la presencia de tres especies de Kainella. El primer registro corresponde a Kainella sp. nov. A en el Miembro Alfarcito de la Formación Santa Rosita, $100 \mathrm{~m}$ por arriba del último registro de Jujuyaspis keideli. Esta especie es inmediatamente sucedida por $K$. meridionalis, que se extiende a través de aproximadamente $240 \mathrm{~m}$ de la columna sedimentaria. La Biozona de K. meridionalis se reconoce también en las localidades de Iruya (Quebrada Colorada y Río Bocoyá) y La Caldera. K. andina y K. meridionalis se registran dentro de la zona de conodontes de C. angulatus y se incluyen en la parte superior del Tr1 (Tremadociano inferior).

Existe un cierto grado de incertidumbre con respecto a la relación entre la Biozona de K. andina y la de $K$. meridionalis, ya que estas especies aún no han sido documentadas en una misma sección medida. Sin embargo, existe una clara relación evolutiva entre las distintas especies descriptas en este trabajo. En este sentido, las especies progresivamente más jóvenes manifiestan un aumento progresivo en el ancho de las áreas intraoculares, un aumento en el número de anillos axiales en el pigidio y un cambio en su forma (de rectos a sinuosos). Dicha relación puede ser resumida de esta manera:

K. morena/K. andina $\rightarrow$ K. sp. nov A/K. sp. nov. B $\rightarrow$ K. meridionalis/K. teiichii.

Cabe destacar que un muestreo preliminar realizado en la sección de Azul Pampa permite anticipar la presencia de $K$. meridionalis y, como fue enunciado 
previamente, se documentan también K. morena y $K$. andina. Sin embargo, es aún prematuro establecer el rango de las especies y la extensión de las biozonas en esta localidad.

Biozona de Kainella teiichii nov. La base de esta biozona está determinada por el primer registro de $K$. teiichii en la sección de Arenal, que se propone como estratotipo. Esta biozona se extiende en esta localidad a través de aproximadamente $50 \mathrm{~m}$ en la columna sedimentaria y su límite superior está determinado por el registro de una asociación compuesta por Pseudokainella keideli, Bienvillia tetragonalis y Peltocare sp. (figura 4).

La edad de K. teiichii puede ser precisada a partir del registro de esta especie en el tramo medio-superior de la Formación Cardonal, y el tramo inferior de la Formación Saladillo en Pascha (K. meridionalis de Tortello y Rao, 2000; ver Vaccari y Waisfeld, 2010). Vaccari y Waisfeld (2010) indicaron que la parte inferior corresponde a la parte alta de la Biozona de Cordylodus angulatus, mientras que la parte superior, ya en la Formación Saladillo, está asociada a conodontes de edad tremadociana media temprana. Esta misma edad fue reportada por Ortega y Albanesi $(2002,2003)$ sobre la base del registro de la Biozona de Bryograptus (Tremadociano medio temprano, Tr2) presente en la parte inferior de la Formación Saladillo. La Biozona de $K$. teiichii se reconoce, además, en las áreas de Iruya y, probablemente, Alfarcito por el registro de esta especie.

En síntesis, de acuerdo a esta reevaluación, la clásica Biozona de "K. meridionalis" de Harrington y Leanza (1957) del noroeste argentino incluye un rango amplio que puede ser subdividido en tres biozonas distintas sobre la base del registro de las diferentes especies de Kainella: K. andina y K. meridionalis del Tremadociano inferior (parte superior del Tr1), y $K$. teiichii que se extendería hasta la base del Tremadociano medio (Tr2) (figura 5).

\section{Correlación intercontinental}

Como se ha mencionado previamente, las especies de Kainella han sido utilizadas para la correlación intercontinental. Harrington y Kay (1951) establecieron la correlación de parte de la fauna del Grupo Güéjar (Tremadociano-Arenigiano), Caño Guapayito, Sierra de La Macarena, en Colombia, con la Biozona de K. meridionalis, en base a la presencia de K. colombiana. Sin embargo, se propone excluir esta especie del género (ver Vaccari y Waisfeld, 2010), reubicándola tentativamente en el género Naustia Ludvigsen, 1982. Esto reviste importancia bioestratigráfica, ya que las especies más cercanas con las que hemos comparado Naustia? colombiana son de edad fu-

\begin{tabular}{|c|c|c|}
\hline \multirow{4}{*}{ 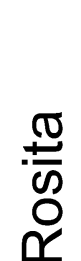 } & \multirow{2}{*}{ Mb. Rupasca } & B. tetragonalis \\
\hline & & K. teiichii \\
\hline & \multirow{3}{*}{ Mb. Alfarcito } & K. meridionalis \\
\hline & & K. andina ? \\
\hline \multirow{4}{*}{ 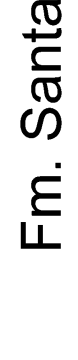 } & & J. keideli \\
\hline & $\begin{array}{l}\text { Mb. Pico } \\
\text { de Halcón }\end{array}$ & $\begin{array}{c}\text { hiatus } \\
\text { N. f. argentina }\end{array}$ \\
\hline & $\begin{array}{l}\text { Mb. Casa } \\
\text { Colorada }\end{array}$ & N. f. argentina \\
\hline & Mb. Tilcara & Sin trilobites \\
\hline
\end{tabular}

Figura 4. Biozonas reconocidas al este de la Quebrada de Humahuaca, sector oriental de la Cordillera Oriental / biozones recognized East of Quebrada de Humahuaca, eastern area of the Cordillera Oriental.

rongiana. Por lo tanto, no se descarta que los estratos de Grupo Gúéjar que contienen esta especie puedan ser referibles al Furongiano.

Jell (1985) correlacionó la fauna de la Formación Digger Island (Victoria, Australia) con la Zona de K. meridionalis por la presencia de Leiostegium douglasi Harrington, Australoharpes y Pseudokainella. En Argentina, Leiostegium douglasi ha sido reconocida en las secciones de la Sierra de Cajas en la Biozona de Neoparabolina frequens argentina (Furongiano) (Benedetto, 1977; Tortello et al., 1999), y en la "Biozona de Kainella meridionalis" (Harrington y Leanza, 1957; Aceñolaza, 1968), para la Sierra de Cajas y Pueblo Viejo (Salta). En la Faja Eruptiva de la Puna Oriental hemos reconocido esta especie en las formaciones Taique y Potrerillos en la Biozona de N. frequens argentina y en la Biozona de Jujuyaspis keideli respectivamente, y en la Formación Las Vicuñas en la Faja Eruptiva de la Puna Occidental (Vaccari y Waisfeld, 2000). Por otra parte, Peng (1990a, p. 20) sugirió que Pseudokainella diggerensis Jell de la Formación Digger Island debería ser reubicada en Apatokephalops Lu, 1975, y correlacionó esta fauna con la Biozona de Onychopyge-Hysterolenus (Peng, 1990b, p.69). Por lo tanto, su registro sería algo más antiguo que el de la fauna de Kainella.

Wright et al. (1994), sobre la base de la presencia de K. meridionalis (=Kainella sp. en este trabajo), correlacionó su fauna 3 de la Summit Limestone de Nueva Zelanda (Terreno Takata), con parte de la "Biozona de K. meridionalis" en Argentina. Por la posición de esta fauna, por encima de la Biozona de Cordylodus angulatus, podría correlacionarse, parcialmente, con la Biozona de Kainella teiichii. 


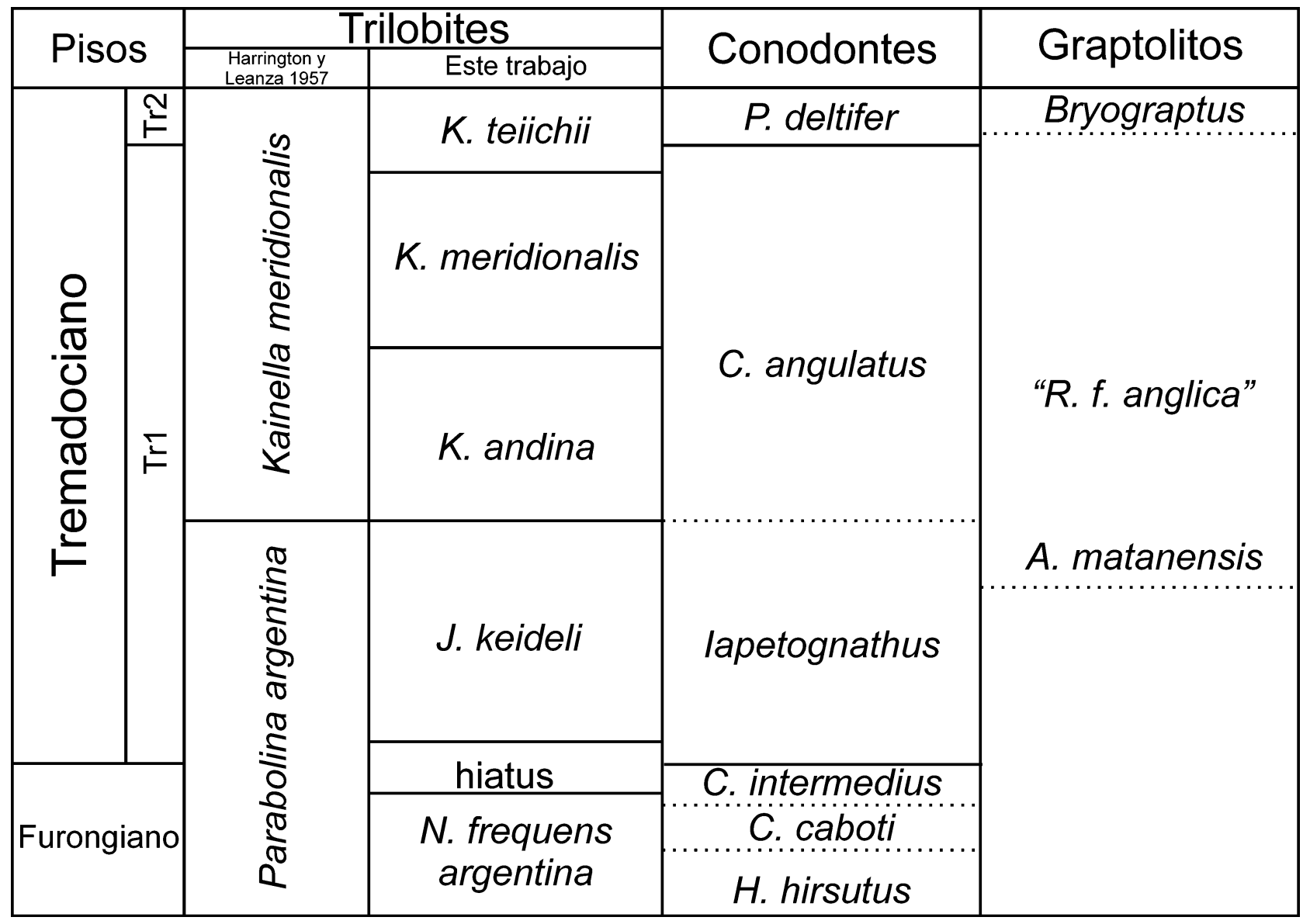

Figura 5. Esquema bioestratigráfico integrado propuesto para el Tremadociano de la Cuenca Andina Central. Información de conodontes y graptolitos tomada de Rao y Hünicken (1995) y Ortega y Albanesi (2005) / integrated biostratigraphic framework for the Tremadocian of the Central Andean Basin. Information on conodonts and graptolites from Rao and Hünicken (1995), and Ortega and Albanesi (2005).

Kim y Choi (2000) definieron la Biozona de Kainella, integrada por K. euryarchis, y Leiostegium sp. en la base del Miembro Chommal de la Formación Mungog (área de Yeongweol, Korea), y la refirieron al Tremadociano medio por correlación con la Zona de K. meridionalis de Argentina, y con las biozonas de Kainella-Leiostegium y de Tesselacauda de América del Norte.

Ross et al. (1997) correlacionaron la Biozona de Kainella-Leiostegium (=Biozona D, Hintze, 1953), que marca la base del Stairsiano de la Serie Ibexiana en el Great Basin, con la Biozona de K. meridionalis en Argentina. De acuerdo a Ross et al. (1997), la Biozona de Kainella-Leiostegium en América del Norte está restringida a un intervalo relativamente corto que se corresponde al intervalo de baja diversidad de las zonas de condontes ("Low Diversity Interval"). En particular, los registros de Kainella están restringidos a la Zona E (Biozona de Tesselacauda) con K. stenorachis (=K. kindlei Dean, 1989, p. 34) y a la Biozona de Kainella-Leiostegium (Zona D). Sin embargo, el rango de Leiostegium es significativamente más amplio. A moAMEGHINIANA 47 (3), 2010 do de ejemplo, la especie norteamericana Leiostegium valmyensis (Lochman) ha sido reconocida en la Biozona G1 (=Hintzeia celsuora) en las formaciones Valmy (Nevada) y Deadwood (Montana) y en la Zona F (=Rossaspis superciliosa) en la Formación Wilcox Pass (Dean, 1989, p. 30).

Por otra parte, Vaccari (2001) describió Leiostegium precordilleranus Vaccari de la Formación San Juan en la Precordillera Argentina. Este autor reconoció esta especie en el intervalo que comprende las Biozona de Paltodus deltifer y Prioniodus elegans (Tremadociano tardío-Floiano temprano). También Kim y Choi (2000) advirtieron el amplio rango estratigráfico de Leiostegium en China, Australia, América del Sur y América del Norte. Así queda demostrado que el registro de Leiostegium comprende un intervalo que va desde el Cámbrico tardío alto hasta el Floiano temprano.

Como se ha puntualizado, los registros de Kainella en Argentina comprenden desde la Biozona de Cordylodus angulatus (Biozona de K. andina/Biozona de K. meridionalis) hasta la Biozona de Bryograptus del Tremadociano medio bajo (posiblemente Biozona de 
K. teiichii). De esta manera, por su asociación de conodontes del Low Diversity Interval, la Biozona de Kainella-Leiostegium de América del Norte se correlacionaría tentativamente con parte de la Biozona de $K$. teiichii, o podría ser aún más joven.

En síntesis, las correlaciones mencionadas con las sucesiones argentinas han tomado en consideración un rango amplio que corresponde en realidad al registro completo del género, equivalente a la "Fauna de Kainella" o a la Biozona de "Kainella meridionalis" de Harrington y Leanza (1957). La subdivisión de la misma que se propone en esta contribución, brinda un marco de referencia para establecer correlaciones mas precisas en el futuro. Una correlación intercontinental más ajustada sólo sería posible calibrando el rango de cada especie y precisando su edad sobre la base de especies de distribución cosmopolita.

\section{Agradecimientos}

Queremos dejar constancia de nuestro agradecimiento a. R. Suárez Soruco del Museo de Historia Natural de Cochabamba por su invitación a estudiar las faunas de trilobites del Ordovícico de Bolivia. Asimismo, agradecemos a F. Tortello la información sobre la posición estratigráfica del material de la quebrada Lampazar. Los autores agradecen el financiamiento de CONICET (PIP $\mathrm{N}^{\circ}$ 5599, 6348 y 112200801 01994) y ANPCyT-FONCyT (PICT $2004 \mathrm{~N}^{\circ}$ 21857 y PICT 2006 No 1272).

\section{Bibliografía}

Aceñolaza, F.G. 1968. Geología estratigráfica de la región de la Sierra de Cajas, Departamento. Humahuaca (Provincia de Jujuy). Revista de la Asociación Geológica Argentina 23: 207-222.

Aceñolaza, F.G. y Aceñolaza, G.F. 1992. The genus Jujuyaspis as a world reference fossil for the Cambrian-Ordovician boundary. En: B.D. Webby y J.R. Laurie (eds.), Global perspectives on Ordovician Geology Balkema, Rotterdam., pp. 115-120.

Aceñolaza, G.F., Aráoz, L., Vergel, M.M., Tortello F. y Nieva, S.M. 2003. Paleontology and biostratigraphy of the Lower Ordovician strata cropping out at the Abra de Zenta (Cordillera Oriental, Jujuy and Salta provinces), NW Argentina. En: G.L. Albanesi, M.S. Beresi y S.H. Peralta (eds.), Ordovician from the Andes. INSUGEO, Serie de Correlación Geológica, 17: 23-28.

Albanesi, G.L. y Aceñolaza, G.F. 2005. Conodontes de la Formación Rupasca (Ordovícico Inferior) en el Angosto de Chucalezna, Cordillera Oriental de Jujuy: nuevos elementos bioestratigráficos para una localidad clásica del noroeste argentino. Ameghiniana 42: 295-310.

Astini, R.A. 2003. The Ordovician Proto-Andean basins. En: J.L. Benedetto (ed.), Ordovician fossils of Argentina. Secretaría de Ciencias y Tecnología, Universidad Nacional de Córdoba, pp. $1-74$

Astini, R.A. 2005. Las unidades sedimentarias que reposan en no concordancia sobre el granito rojo en el angosto de la Quesera (Cordillera Oriental, Salta) y su interpretación estratigráfica: una revisión crítica a más 60 años de los trabajos pioneros de J. Keidel. Revista de la Asociación Geológica Argentina 60: 513-523.

Baldis, B.A., González, S.B. y Pérez, V.E. 1984. Trilobites tremadocianos de la Formación Negritos (Perforación La Heliera), Llanos de Colombia. $3^{\circ}$ Congreso Latinoamericano de Paleontología (Oaxtepec), Memoria 3: 28-41.
Barnes, C.R. 1988. The proponed Cambrian-Ordovician Boundary stratotype and point (GSSP) in Western Newfoundlan, Canada. Geological Magazine 125: 381-414.

Benedetto, J.L. 1977. Algunas consideraciones acerca de la posición del límite Cambro-Ordovícico en América del Sur. Geos 23: 3-11.

Benedetto, J.L. 2005. Hacia un esquema bioestratigráfico de alta resolución para el Cámbrico Superior - Ordovícico del noroeste de Argentina, basado en filozonas de braquiópodos rhynchonelliformes. $16^{\circ}$ Congreso Geológico Argentino (La Plata), Acta 3: 371-378.

Benedetto, J.L. 2007. New Upper Cambrian-Tremadoc rhynchonelliformean brachiopods from Northwestern Argentina: Evolutionary trends and early diversification of plectorthoideans in the Andean Gondwana. Journal of Paleontology 81: 261-285.

Bergström, S.M., Chen, X., Gutiérrez-Marco, J.C. y Dronov, A. 2009. The new chronostratigraphic classification of the Ordovician System and its relations to major regional series and stages and to $\delta^{13} \mathrm{C}$ chemostratigraphy. Lethaia 42 : 97-107.

Branisa, L. 1965. Los fósiles guías de Bolivia. Boletín del Servicio Geológico de Bolivia 6: 1-82.

Buatois, L.A. y Mángano, M.G. 2003. Sedimentary facies, depositional evolution of the Upper Cambrian-Lower Ordovician Santa Rosita Formation in Northwest Argentina. Journal of South America Earth Sciences 16: 343-363.

Buatois, L.A., Moya, M.C., Mángano, M.G. y Malanca, S. 2003. Paleoenvironmental and sequence stratigraphic framework of the Cambrian-Ordovician transition in the Angosto del Moreno Area, Northwest Argentina. En: G.L. Albanesi, M.S. Beresi y S.H. Peralta (eds.), Ordovician from the Andes. INSUGEO Serie de Correlación Geológica 17: 397-401.

Buatois, L.A., Zeballo, F.J., Albanesi, G.L., Ortega, G., Vaccari, N.E. y Mángano, M.G. 2006. Depositional environments and stratigraphy of the Cambrian-Tremadocian Santa Rosita Formation at the Alfarcito area, Cordillera Oriental, Argentina: Integration of biostratigraphic data within a sequence stratigraphic framework. Latin American Journal of Sedimentology and Basin Analysis 13: 65-94.

Cooper, R.A., Nowlan, E.S. y Williams, S.H., 2001. Global stratotype section and point for the base of the Ordovician System. Episodes 24: 19-28.

Dean, W.T. 1989. Trilobites from the Survey Peak, Outram and Skoki Formations (Upper Cambrian-Lower Ordovician) at Wilcox Pass, Jasper National Park, Alberta. Geological Survey of Canada, Bulletin 389: 1-141.

Egenhoff, S.O., Maletz, J. y Erdtmann, B.-D. 2004. Lower Ordovician graptolite zonation and lithofacies of southern Bolivia: relevance for palaeogeographic interpretations. Geological Magazine 141: 287-299.

Erdtmann, B.D, Kley, J., Müller y Jacobshagen, V. 1995. Ordovician basin dynamics and new graptolite data from Tarija region, eastern Cordillera, south Bolivia. En: J.D. Cooper, M.L. Droser y S.C. Finney (eds.), Ordovician-Odysssey. Society of Economic Paleontologist and Mineralogists, Pacific Section, Book 77, p. 69-73. Las Vegas

Esteban, S. y Tortello, M.F. 2007. Latest Cambrian sedimentary settings and trilobite faunas from the western Cordillera Oriental, Argentina. Memoirs of the Association of the Australasian Paleontologists 34: 431-460.

Fernández, R.I. 1985. Bioestratigrafía cambro-ordovícica del tramo superior de la quebrada de Humahuaca, Provincia de Jujuy, Argentina. $4^{\circ}$ Congreso Geológico Chileno (Antofagasta), Acta 1: 284-308.

Gómez Martínez, E., Sánchez Lopez, M., De Simona, H. y Heit, F. 2002. Paleoambientes reconocidos en la formación Saladillo (Ordovícico) en el sudoeste de la Cordillera Oriental. $15^{\circ}$ Congreso Geológico Argentino (Calafate), Actas 1:738-741.

Harrington, H.J. 1938. Sobre las faunas del Ordoviciano inferior del norte Argentino. Revista del Museo de La Plata, Sección Paleontología 4: 109-289. 
Harrington, H.J. y Leanza, A. 1957. Ordovician trilobites of Argentina. University of Kansas Press, Lawrence, 276 pp.

Harrington, H.J. y Kay, M. 1951. Cambrian and Ordovician faunas of Eastern Colombia. Journal of Paleontology 25: 655-668.

Hintze, L.F. 1953. Lower Ordovician trilobites from western Utah and eastern Nevada. Utah Geological and Mineralogical Survey Bulletin 48: 1-249.

Jell, P.A. 1985. Tremadoc trilobites of the Digger Island Formation,Waratah Bay, Victoria. Memoirs of the Museum of Victoria 46: 53-88.

Kim, D.H. y Choi, D.K. 2000. Lithostratigraphy and biostratigraphy of the Mungok Formation (Lower Ordovician), Yungwol, Korea. Geoscience Journal 4: 301-311.

Kobayashi, T. 1935. The Kainella fauna of the basal Ordovicician age found in Argentina. Japanese Journal Geology and Geography 12: 59-67.

Kobayashi, T. 1937. The Cambro-Ordovician shelly faunas of South America. Journal of the Faculty of Science, Imperial University of Tokyo 4: 369-522.

Lu, Y-H. 1975. Ordovician trilobite faunas of central and southwest China. Paleontologia Sinica 11: 1-460.

Ludvigsen, R. 1982. Upper Cambrian to Lower Ordovician trilobite biostratigraphy of the Rabbitkettle Formation, western District of Mackenzie. Life Sciencies Contributions, Royal Ontario Museum 134: 1-188.

Mángano, M.G. y Buatois, L.A. 2004. Integración de estratigrafía secuencial, sedimentología e icnología para un análisis cronoestratigráfico del Paleozoico inferior del noroeste argentino. Revista de la Asociación Geológica Argentina 59: 273-280.

Méndez, V. 1973. Geología de la comarca de Mina "Aguilar" y alrededores, Departamento Humahuaca (Provincia de Jujuy). Revista de la Asociación Geológica Argentina 28: 319-330.

Miller, J.F., Ethington, R.L., Evans, K.R., Holmer, L.E., Lochid, J.D., Popov, L.E., Repetski, J.E., Ripperdan, R.L. y Taylor, J.F. 2006. Proposed stratotype for the base of the highest Cambrian stage at the first appearance datum of Cordylodus andresi, Lawson Cove section, Utah, USA. Palaeoworld 15: 384-405.

Moya, M.C. 1988. Lower Ordovician in the Southern part of the Argentine Eastern Cordillera. Lecture Notes in Earth Sciences 17: 55-69.

Moya, M.C. 1998. El Paleozoico inferior en la sierra de Mojotoro, Salta - Jujuy. Revista de la Asociación Geológica Argentina 53: 219238

Moya M.C. y Albanesi, G.L. 2000. New stratigraphic section to define the Cambrian-Ordovician boundary in Eastern Cordillera, northwest Argentina. En: G.F. Aceñolaza y S. Peralta (eds.), Cambrian of the southern edge. INSUGEO, Miscelánea 6: 114-116.

Moya, M.C. y Monteros, J.A. 2000. El Angosto del Moreno (Cordillera Oriental argentina), un área clave para analizar el límite Cambro-Ordovícico y la discordancia Iruya. $14^{\circ}$ Congreso Geológico Boliviano (La Paz), Actas: 142-147.

Moya, M.C., Malanca, S., Monteros, J.A. y Cuerda, A.J. 1994. Bioestratigrafía del Ordovícico Inferior en la Cordillera Oriental Argentina basada en graptolitos. Revista Española de Paleontología 9: 91-104.

Moya, M.C., Malanca, S., Monteros, J. A., Albanesi, G.L., Ortega, G. y Buatois, L. 2003. Late Cambrian-Tremadocian faunas and events from Angosto del Moreno Section, Eastern Cordillera, Argentina. En: G.L. Albanesi, M.S. Beresi y S.H. Peralta (eds.), Ordovician from the Andes. INSUGEO, Serie de Correlación Geológica 17: 439-444.

Müller, J., Maletz, J., Egenhoff, S. y Erdtmann, B.D. 1996. Turbiditas Caradocianas - ?Ashgillianas inferiores en la Cordillera Oriental del sur de Bolivia: Implicaciones cinemáticas. $12^{\circ}$ Congreso Geológico de Bolivia (Tarija), Acta 1: 747-753.

Nikolaisen, F. y Henningsmoen, G. 1985. Upper Cambrian and lower Tremadoc olenid trilobites from the Digermul peninsula, Finnmark, northern Norway. Norges Geologiske Undersøkelse Bulletin 400: 1-49.
Ortega, G. y Albanesi, G.L. 2002. Bioestratigrafía de graptolitos y conodontes del Tremadociano Tardío de la Cordillera Oriental Argentina. $15^{\circ}$ Congreso Geológico Argentino y $5^{\circ}$ Congreso de Exploración de Hidrocarburos (Calafate), Acta 1: 542-547.

Ortega, G. y Albanesi, G.L. 2003. Late Tremadoc graptolite sequence and conodonts from the Parcha area, Eastern Cordillera, Argentina. En: G. Ortega y G.F. Aceñolaza (eds.). Proceedings of the $7^{\circ}$ Internacional Graptolite Conference. INSUGEO, Serie Correlación Geológica 18: 79-86.

Ortega, G. y Ǎlbanesi, G.L. 2005. Tremadocian graptolite-conodont biostratigraphy of the South American Gondwana margin (Eastern Cordillera, NW Argentina). Geologica Acta 3: 355371.

Přribyl, A. y Vaněk, J. 1980. Ordovician trilobites of Bolivia. Rozpravy Československé Akademie Vèd, Řada Matematických a Prírodních veedy 90: 1-90.

Peng, S. 1990a. Tremadoc stratigraphy and trilobite faunas of northwestern Hunan. 1, Trilobites from the Nantsinkwan Formation of the Yangtze Platform. Beringeria 2: 3-53

Peng, S. 1990b. Tremadoc stratigraphy and trilobite faunas of northwestern Hunan. 2. Trilobites from the Panjiazui Formation and the Madaoyu Formation in Jiangnan Slope Belt. Beringeria 2: 55-171.

Rao, R.I. 1999. Los conodontes Cambro-Ordovícicos de la sierra de Cajas y del Espinazo del Diablo, Cordillera Oriental, República Argentina. Revista Española de Micropaleontología 31: 2351.

Rao, R.I. y Hünicken, M.A. 1995. Conodont biostratigraphy of the Cambrian-Ordovician boundary in northwestern Argentina. En: J.D. Cooper, M.L. Droser y S.C. Finney (eds.), OrdovicianOdysssey. Society of Economic Paleontologist and Mineralogists, Pacific Section, Book 77, pp. 125-128. Las Vegas

Rao, R.I. y Tortello, M.F. 1998. Tremadoc conodonts and trilobites from the Cardonal Formation, Incamayo Creek, Salta Province, northwestern Argentina. Palaeontologia Polonica 58: 31-45.

Ross R.J.Jr, Hintze, L.F., Ethington R.L., Miller, J.F., Taylor, M.E. y Repetsky J.E., 1997. The Ibexian, Lowermost Series in The North American Ordovician. U.S. Geological Survey Professional Paper 1579-A: 1-50.

Rubinstein, C.V., Toro, B.A. y Waisfeld, B.G. 1999. Acritarch biostratigraphy of the upper Tremadoc-Arenig of the Eastern Cordillera, northwestern Argentina: relationships with graptolite and trilobite fauna. Bolletino della Società Paleontologica Italiana 38: 267- 286.

Salas, M.J., Vannier, J. y Willians, M., 2007. Early Ordovician ostracods from Argentina: Their bearing on the origin of binodicope and palaeocope clades. Journal of Paleontology 81: 13841395.

Steinmann, G. y Hoek, H. 1912. Das Silur und Cambrium des Hochlandes von Bolivia und ihre Fauna. Neues Jahrbuch für Mineralogie, Geologie und Paläontologie 34: 176-252.

Suárez Soruco, R. 1975. Nuevos trilobites del Tremadociano inferior (Ordovícico) del sur de Bolivia. Revista Técnica, Yacimientos Petrolíferos Fiscales Bolivianos 4: 129-146.

Toro, B.A, de la Puente, G.S. y Rubinstein, C.V. 2010. New graptolite, chitinozoan and acritarch records from the PaschaIncamayo area, Cordillera Oriental, Argentina. Comptes Rendus Palevol 9: 23-30.

Tortello, M.F. y Aceñolaza, G.F. 1993. Trilobites agnóstidos del límite Cámbrico-Ordovícico de la Formación Lampazar, Sierra de Cajas, Provincia de Jujuy, Argentina. Revista Española de Paleontología 8: 177-184.

Tortello, M.F. y Clarkson, E.N.K. 2003. Ontogeny of the Early Ordovician olenid trilobite Jujuyaspi keideli Kobayashi from northwestern Argentina. Ameghiniana 40: 257-275.

Tortello, M.F y Esteban, S.B. 2003. Trilobites del Cámbrico tardío de la Formación Lampazar (Sierra de Cajas, Jujuy, Argentina). Implicancias bioestratigráficas y paleoambientales. Ameghiniana 40: 323-344. 
Tortello, M.F., Esteban, S.B. y Aceñolaza, G.F. 2002. Trilobites from the base of the Ordovician System in Northwestern Argentina. En: F.G. Aceñolaza (ed.), Aspects of the Ordovician System in Argentina. INSUGEO, Serie de Correlación Geológica 16: 131-142.

Tortello, M.F., Rabano, I., Rao, R.I. y Aceñolaza, F.G. 1999. Los trilobites de la transición Cámbrico-Ordovícico en la Quebrada Amarilla (Sierra de Cajas, Jujuy, Argentina). Boletín Geológico y Minero 110: 555-572.

Tortello, M.F. y Rao, R.I. 2000. Trilobites y conodontes del Ordovícico temprano del Angosto de Lampazar (provincia de Salta, Argentina). Boletín Geológico y Minero 111: 61-84.

Vaccari, N.E. 2001. Trilobites de la Formación San Juan (Ordovícico inferior), Precordillera Argentina. Ameghiniana 38: 331-348.

Vaccari, N.E., y Waisfeld, B.G. 2000. Trilobites tremadocianos de la Formación Las Vicuñas, Puna occidental, Provincia de Salta, Argentina. $14^{\circ}$ Congreso Geológico Boliviano (La Paz), Memorias 1: 165-169.

Vaccari, N.E., y Waisfeld, B.G., 2010. Kainella Walcott, 1925 (Trilobita, Ordovicico Temprano) en el Noroeste de Argentina y Sur de Bolivia. Paleontología Sistemática. Ameghiniana 47: 273-292.

Vaccari, N.E., Waisfeld B.G. y Smith, L., 2006. Trilobites de la Formación Iscayachi (sur de Bolivia). Consideraciones bioestratigráficas. $9^{\circ}$ Congreso Argentino de Paleontología y Bioestratigrafía (Córdoba), Resúmenes 1: 168

Vaccari, N.E., Waisfeld, B.G. y Balseiro, D. 2008. Una discontinuidad estratigráfica en el límite furnigiano-tremadociano de la Formación Santa Rosita en la quebrada de Humahuaca, Cordillera Oriental, Argentina. $17^{\circ}$ Congreso Geológico Argentino (San Salvador de Jujuy), Actas 3: 1059-1060.

Vergel, M.del M., Aceñolaza, G.F. y Aráoz, L. 2007. La Formación Casa Colorada en la quebrada de Moya (Cambro-Ordovícico): aportes a la cronoestratigrafía de una localidad clásica de la Cordillera Oriental de Jujuy (Argentina). Ameghiniana 44: 621630.

Waisfeld, B.G. y Vaccari, N.E. 2003. Trilobites. En: J.L. Benedetto (ed.), Ordovician Fossils of Argentina. Secretaría de Ciencia y Tecnología, Universidad Nacional de Córdoba, pp. 295-410, Córdoba.

Waisfeld, B.G. y Vaccari, N.E. 2006. Revisión de la Biozona de Ogygiocaris araiorhachis (Trilobita, Tremadociano tardío) en la región de Pascha-Incamayo, Cordillera Oriental Argentina. Parte 2: Sistemática. Ameghiniana 43: 729-744.
Waisfeld, B.G., Vaccari, N.E., Toro, B.A., Rubinstein, C.V. y Astini, R.A. 2006. Revisión de la Biozona de Ogygiocaris araiorhachis (Trilobita, Tremadociano tardío) en la región de Pascha-Incamayo, Cordillera Oriental Argentina. Parte 1: Bioestratigrafía. Ameghiniana 43: 717-728.

Walcott, C.D. 1925. Cambrian geology and paleontology. Cambrian and Ozarkian trilobites. Smithsonian Miscellaneous Colletions 75: 61-146.

Wright, A.J., Cooper, R.A., y Simes, J.E. 1994. Cambrian and Ordovician faunas and stratigraphy, Mt Patriarch, New Zealand. New Zealand Journal of Geology and Geophysics 37: 437476.

Zeballo, F.J. y Albanesi, G.L. 2008. Conodontes cámbricos asociados a los trilobites Jujuyaspis keideli y Parabolina f. argentina en la quebrada de Humahuaca, Jujuy, Argentina. $17^{\circ}$ Congreso Geológico Argentino (San Salvador de Jujuy), Actas 1: 376-377.

Zeballo, F.J. y Albanesi, G.L. 2009. Conodontes cámbricos y Jujuyaspis keideli Kobayashi (Trilobita) en el Miembro Alfarcito de la Formación Santa Rosita, quebrada de Humahuaca, Cordillera Oriental de Jujuy. Ameghiniana 46: 537-556.

Zeballo, F.J., Albanesi, G.L. y Ortega, G. 2005. Conodontos y graptolitos de las formaciones Alfarcito y Rupasca (Tremadociano) en el área de Alfarcito, Tilcara, Cordillera Oriental de Jujuy, Argentina. Parte 1: Bioestratigrafía. Ameghiniana 42: 39-46.

Zeballo, F.J., Albanesi, G.L. y Ortega, G. 2008. New late Tremadocian (Early Ordovician) conodont and graptolite records from the southern South American Gondwana margin (Eastern Cordillera, Argentina). Geologica Acta 6: 127-141

Recibido: 28 de mayo de 2008 . Aceptado: 26 de mayo de 2010. 